\title{
FLAVONÓIDES, NORISOPRENÓIDES E OUTROS TERPENOS DAS FOLHAS DE Tapirira guianensis
}

\author{
Suzimone de J. Correia \\ Departamento de Química e Exatas, Universidade Estadual do Sudoeste da Bahia, 45206-190 Jequié - BA, Brasil \\ Jorge M. David* e Eliezer P. da Silva \\ Instituto de Química, Universidade Federal da Bahia, 40170-290 Salvador - BA, Brasil \\ Juceni P. David \\ Faculdade de Farmácia, Universidade Federal da Bahia, 40170-290 Salvador - BA, Brasil \\ Lucia M. X. Lopes \\ Instituto de Química de Araraquara, Universidade Estadual Paulista,14801-970 Araraquara - SP, Brasil \\ Maria Lenise S. Guedes \\ Instituto de Biologia, Universidade Federal da Bahia, 40170-290, Salvador - BA, Brasil
}

Recebido em 21/2/08; aceito em 30/6/08; publicado na web em 31/10/08

\begin{abstract}
FLAVONOIDS, NORISOPRENOIDS AND OTHER TERPENES FROM LEAVES OF Tapirira guianensis. From hexane fraction of methanol extract of leaves of Tapirira guianensis (Anacardiaceae) were obtained lupeol, 24-methylenecycloartan-3-ol, phytol, $\alpha$-amyrin, $\beta$-amyrin, sitosterol, sitostenone, glycosyl sitosterol, as well as sitosterol esterified with palmitic and stearic acids. Phytol, $\alpha$-amyrin and $\beta$-amyrin esterified with fatty acids were also identified from same extract. The EtOAc extract besides the norisoprenoids $(6 S, 7 E, 9 S)$-6,9-dihydroxy-megastigma-4,7-dien-3-one 9-O- $\beta$-glucopyranoside and (6S,7E,9R)-6,9-dihydroxymegastigma-4,7-dien-3-one 9-O- $\beta$-glucopyranoside also afforded kaempferol 3-O-rhamnoside, kaempferol 3-O-arabinofuranoside, quercetin 3-O-rhamnoside, and kaempferol. The structural elucidation of isolated compounds were based on UV, IR, MS, ${ }^{1} \mathrm{H}$ and ${ }^{13} \mathrm{C}$ NMR data analysis.
\end{abstract}

Keywords: Tapirira guianensis; norisoprenoides; fatty acid esters of triterpenes.

\section{INTRODUÇÃO}

Anacardiaceae é uma família constituída por 76 gêneros e 600 espécies. Cerca de $25 \%$ dos gêneros dessa família são conhecidos por possuírem espécies reconhecidas como tóxicas e causadoras de dermatite de contato severa. De modo geral, as espécies venenosas desta família estão restritas às tribos Anacardieae, Rhoeae e Semecarpeae. ${ }^{1}$ O gênero Tapirira é composto de aproximadamente 15 espécies que ocorrem principalmente na América do Sul. Tapirira guianensis Aubl. é uma árvore cuja altura varia de 8-14 m e que tem ocorrência em todo território brasileiro, principalmente em terrenos úmidos, em quase todas as formações vegetais. É conhecida popularmente como "pau-pombo" e é utilizada na medicina popular contra lepra, diarreia e sífilis. ${ }^{2,3}$ Espécies da família Anacardiaceae são conhecidas por conterem lipídios fenólicos, compostos distinguidos por suas propriedades vesicantes, que são responsáveis pela toxicidade. ${ }^{4}$ Em estudos anteriores realizados com espécies do gênero foram isolados terpenos, ferulatos e derivados de alquil e alquenil fenóis. ${ }^{2,5}$ No presente trabalho encontra-se descrita a composição química dos extratos hexânico e AcOEt obtida a partir das folhas de um espécime de T. guianensis.

\section{PARTE EXPERIMENTAL}

Os espectros de RMN foram registrados em espectrômetro de RMN Varian Gemini 2000 e Inova 500 (7,05 e 11,74 Tesla), operando a 300 e $500 \mathrm{MHz}\left({ }^{1} \mathrm{H}\right)$ e 75 e $125 \mathrm{MHz}\left({ }^{13} \mathrm{C}\right)$, respectivamente. As aquisições dos espectros foram realizadas a 26 e $30^{\circ} \mathrm{C}$, respectivamente, utilizando-se solventes deuterados $\left(\mathrm{CDCl}_{3}\right.$, metanol- $d_{4}$, piridina- $\left.d_{5}\right)$, com grau de pureza superior a 99,8\%, das marcas Aldrich ${ }^{\circledR}$, Merck $^{\circledR}$

\footnotetext{
*e-mail: jmdavid@ufba.br
}

ou Isotech. Os sinais dos espectros foram registrados com base nos sinais do solvente empregado em relação ao TMS.

Os ésteres metílicos dos ácidos graxos foram identificados por CG-EM, empregando-se cromatógrafos da Varian mod. Saturn II e Hewllett Packard mod. 5970 equipados com coluna DB-5 30,0 m x $0,25 \mathrm{~mm}$, com filme de $0,25 \mu \mathrm{m}$. As condições de operação das análises cromatográficas foram as seguintes: temperatura programada da coluna de $50{ }^{\circ} \mathrm{C}$ por $1 \mathrm{~min}$, velocidade de aquecimento $15^{\circ} \mathrm{C} / \mathrm{min}$ até $250{ }^{\circ} \mathrm{C}$, mantida por $2 \mathrm{~min}$; temperatura do injetor de $200{ }^{\circ} \mathrm{C}$; temperatura da interface de $230^{\circ} \mathrm{C}$; hélio foi empregado como gás de arraste (fluxo $1,1 \mathrm{~mL} / \mathrm{min}$ ). A corrida com detector de massa foi feita na forma scan, com tempo de aquisição de 9 a 40 min; voltagem do filamento de $70 \mathrm{eV}$; voltagem do detector de 1,3 KV; analisador do tipo quadrupolo. Os ésteres metílicos foram identificados através de comparação com os espectros de massas das bibliotecas NIST 62 e NIST 12. Os espectros de absorções no IV foram registrados utilizando-se espectrofotômetros FT-IR Bolmen Hartmann \& Braum, modelo MB100, Nicolet Impact 400 e Nicolet-730 FT-R, empregando-se pastilhas de $\mathrm{KBr}$ ou filme líquido. As rotações ópticas foram medidas em polarímetro Polamat A Carl Zeiss Jena à temperatura ambiente $\left(19-25^{\circ} \mathrm{C}\right)$. Os espectros de massas foram registrados em espectrômetro de massas de baixa resolução FISONS-Modelo VG Platfform II, no modo ESI positivo. Para obtenção das curvas de DC empregou-se espectropolarímetro da marca Jobin Yvon CD6.

\section{Extração e isolamento dos constituintes químicos}

As folhas secas de T. guianensis (2668,0 g) foram maceradas por 48 $\mathrm{h}$, por 3 vezes, seqüencialmente com hexano (5 L) e MeOH (3,5 L). O extrato metanólico (58,0 g) foi ressuspenso em solução de $\mathrm{MeOH}: \mathrm{H}_{2} \mathrm{O}$ (9:1) e, submetido à partição líquido-líquido com solventes de polari- 
dade crescente: hexano e clorofórmio. A fase hidroalcoólica resultante foi submetida à destilação sob pressão reduzida para eliminação do metanol, parcialmente dissolvida em água e particionada com AcOEt (4 x $500 \mathrm{~mL}$ ) e $\mathrm{BuOH}(4$ x $500 \mathrm{~mL})$, sucessivamente. O extrato hexânico foi lavado com MeOH:H $\mathrm{H}_{2} \mathrm{O}(9: 1,4$ x $500 \mathrm{~mL})$. Após eliminação dos solventes, obteve-se a fração metanólica $(22,3 \mathrm{~g})$.

$\mathrm{A}$ fração AcOEt $(8.1 \mathrm{~g})$ foi submetida à $\mathrm{CC}$ empregando-se gel de sílica 60 eluída com misturas de $\mathrm{CHCl}_{3}$-AcOEt e AcOEt-MeOH. Foram coletadas 36 subfrações de $125 \mathrm{~mL}$ cada. Aquela eluída com $\mathrm{CHCl}_{3}$-AcOEt 7:3 (465,3 mg) foi refracionada em CC em sílica gel e, seguida de CCDP empregando $\mathrm{CHCl}_{3}-\mathrm{AcOEt}$ (3:2), fornecendo 1+2 $(23,7 \mathrm{mg})$. A subfração da primeira coluna, eluída com $\mathrm{CHCl}_{3}$-AcOEt 6:4 (118,7 mg), foi também submetida à $\mathrm{CCDP}\left(\mathrm{CHCl}_{3}-\mathrm{MeOH} 4: 1\right)$, levando à obtenção de $\mathbf{3}$ (25,8 mg), $\mathbf{4}$ (37,9 mg) e $\mathbf{5}$ (14,7 mg).

A fração metanólica do extrato hexânico $(22,3 \mathrm{~g})$ foi submetida à $\mathrm{CC}$ em e gel de sílica 60, eluída com misturas de hexano-AcOEt em gradiente crescente de polaridade. Deste fracionamento foram coletadas 36 frações de $250 \mathrm{~mL}$ cada. As frações eluída do extrato original com 5\% de AcOEt $(4,024 \mathrm{~g})$ foram refracionadas em CC, empregando-se misturas de hexano-AcOEt como eluente. Assim, a fração eluída com 5\% de AcOEt $(178,7 \mathrm{mg})$ foi identificada como sendo as substâncias 6-8.

A fração hexânica do extrato metanólico $(11,83 \mathrm{~g})$ foi submetida à CC utilizando-se como suporte gel de sílica 60, eluída com misturas de hexano-AcOEt, da qual foram coletadas 26 subfrações de 250 mL cada. A subfração eluída em hexano-AcOEt 19:1 (2,55 g), após fracionamento em CC, usando como eluente hexano:DCM (95:5), forneceu uma subfração constituída por uma mistura 9-12 (34,1 mg), da subfração eluída com hexano:DCM (9:1) obteve-se uma mistura (78,0 mg) constituída de alcanoatos de $\alpha$-amirinila, de lupeol (13-14) e de $\beta$-sitosterila (15-16). Além dos compostos 9-16, das subfrações da CC empregando-se a fração hexânica do extrato metanólico, foram também sucessivamente isolados das frações desta coluna: sitosterol (17), glicosil sitosterol (18), sitostenona (19), $\alpha$ - e $\beta$-amirina, fitol, lupeol e 24-metilenocicloartan-3-ol (20). ${ }^{5,6}$

\section{Reações de transesterificação}

O procedimento empregado na obtenção dos ésteres graxos dos triterpenos e esteróides consistiu no tratamento das misturas $(5,0 \mathrm{mg})$ com solução de metóxido de sódio $(0,5 \mathrm{M})$ em metanol $(2,0 \mathrm{~mL})$. O isolamento dos ésteres metílicos foi realizado após adição de água destilada à mistura reacional seguido de extração com hexano ou DCM. ${ }^{7}$

(6S,7E,9S)-6,9-diidroxi-megastigma-4,7-dien-3-ona 9-O- $\beta$ glicopiranosídeo (1). Sólido amorfo, amarelo pálido. EM (ESI) 409 $[\mathrm{M}+\mathrm{Na}]^{+}, 387[\mathrm{M}+\mathrm{H}]^{+}$. DC $\left(\mathrm{CH}_{3} \mathrm{OH}, \mathrm{c} 2,610^{-4} \mathrm{M}\right), \Delta \varepsilon(\lambda \mathrm{nm})$ : $20,5^{\circ}$ (240). Dados de RMN de ${ }^{1} \mathrm{H}\left[500 \mathrm{MHz}, \mathrm{CD}_{3} \mathrm{OD}, \delta, J(\mathrm{~Hz})\right]$ : 5,87 (s; H-4); 5,86 (m; H-7); 5,86 (m; H-8); 4,42 (dq; J 3,6 e 6,7 Hz; H-9); 2,60; (dd; $J 2$, 2 e 2,6 Hz; H-2eq); 2, 16 (dd; $J 2,2$ e $J$ 2,6; H-2ax); 1,93 (d; $J 11,0 \mathrm{~Hz} ; J 1,5 \mathrm{~Hz} ; \mathrm{H}-13) ; 1,89$ (s; H-12); 1,29 (d; $J$ $6,7 \mathrm{~Hz} ; \mathrm{H}-10) ; 1,02$ (s; H-11). RMN de ${ }^{13} \mathrm{C}\left(126 \mathrm{MHz}, \mathrm{CD}_{3} \mathrm{OD}, \delta\right)$ : 42,43(C-1); 50,68 (C-2); 201,22 (C-3); 127,16 (C-4); 167,29 (C-5); 80,01(C-6); 131,52 (C-7); 135,26 (C-8); 77,29 (C-9); 21,18 (C-10); 23,42 (C-11); 24,69 (C-12); 19,56 (C-13); 102,71 (C-1'); 75,23(C-2'); 78,34 (C-3'); 71,62 (C-4'); 78,09(C-5'); 62,80 (C-6').

(6S, 7E,9R)-diidroxi-megastigma-4,7-dien-3-ona 9-O- $\beta$ glucopiranosídeo (2). Sólido amorfo, amarelo pálido. EM (ESI) 409 $[\mathrm{M}+\mathrm{Na}]^{+}, 387[\mathrm{M}+\mathrm{H}]^{+}$. DC $\left(\mathrm{CH}_{3} \mathrm{OH}, \mathrm{c} 2,610^{-4} \mathrm{M}\right), \Delta \varepsilon(\lambda \mathrm{nm}):$ $20,5^{\circ}$ (240). Dados de RMN de ${ }^{1} \mathrm{H}\left[500 \mathrm{MHz}, \mathrm{CD}_{3} \mathrm{OD}, \delta, J(\mathrm{~Hz})\right]$ : 5,87 (s; H-4); 5,97 (m; H-7); 5,73 (m; H-8); 4,53 (dq; J 4,0 e 7,8 Hz; H-9); 4,34 (d; $J$ 8,0 Hz; H-1'); 3,85 (dd; $J$ 2,0 e $J$ 11,5 Hz; H-6');
3,26 (dd; $J$ 9,5 e $J$ 8,5 Hz; H-4'); 3,25 (dd; $J$ 9,0 e $J$ 8,5 Hz; H-5'); 3,24 (m, H-3'); 3,18 (m, H-2'); 2,60; (dd; J 2,2 e 2,6 Hz; H-2eq); 2,16 (dd; $J 2,2$ e 2,6 Hz; H-2ax); 1,93 (d; $J 11,0 ; 1,5$ Hz; H-13); 1,89 (s; H-12); 1,13 (d; J 6,5 Hz; H-10); 1,04 (s; H-11); RMN de ${ }^{13} \mathrm{C}(126$ MHz, CD $\mathrm{OD}, \delta$ ): 201,22 (C-3); 167,29 (C-5); 133,75 (C-7); 133,67 (C-8); 127,10 (C-4); 101,22 (C-1'); 80,01(C-6); 78,34 (C-3'); 78,34 (C-5'); 75,23(C-2'); 74,94 (C-9); 71,62 (C-4'); 62,80 (C-6'); 50,68 (C-2); 42,43(C-1); 24,69 (C-12); 23,42 (C-11); 22,20 (C-10); 19,56 (C-13).

Canferol 3- $\alpha$-raminosídeo (3). Sólido amarelo. EM (ESI) em $\mathrm{m} / z$, $455[\mathrm{M}+\mathrm{Na}] . \mathrm{RMN}$ de ${ }^{1} \mathrm{H}\left[300 \mathrm{MHz}, \mathrm{CD}_{3} \mathrm{OD}, \delta, J(\mathrm{~Hz})\right]: 7,75(\mathrm{~d} ; J$ 8,7 Hz; H-2', H-6'); 6,94 (d; J 8,7Hz; H-3', H-5'); 6,34 (sl; H-8); 6, 17 (sl; H-6); 5,36 (sl; H-1"); 4,23 (m; H-2"); 3,73 (m; H-3")) 3,41(m; H-5"); 3,37(m; H-4"); 0,92 (d; J 5,1 Hz ; H-6"). RMN de ${ }^{13} \mathrm{C}(75$ MHz, CD $\mathrm{OD}, \delta$ ): 179,33 (C-4); 166,62 (C-7); 163, 12 (C-5); 161,56 (C-4'); 158,84 (C-9); 158,56 (C-2); 136,11 (C-3); 131,85 (C-2', C-6'); 122,59 (C-1'); 116,52 (C-3', C-5'); 105,67 (C-10); 103,46 (C-1"); 100,08 (C-6); 94,93 (C-8); 73,15 (C-4"); 72,08 (C-3"); 72,01 (C-2"); 71,90 (C-5"); 17,65 ( CH$\left._{3}\right)$.

Canferol 3- $\alpha$-arabinofuranosídeo (4). Sólido amarelo; EM (ESI) $[\mathrm{M}+\mathrm{Na}]^{+} \mathrm{em} m / z$ 441. RMN de ${ }^{1} \mathrm{H}\left[300 \mathrm{MHz}, \mathrm{CD}_{3} \mathrm{OD}, \delta, J(\mathrm{~Hz})\right]: 6,18$ (d; $J$ 1,2 Hz; H-6); 6,39 (d; $J$ 8,8 Hz; H-8); 6,94 (d; $J$ 8,9 Hz; H-3' e H-5'); 7,94 (d; $J$ 8,9 Hz; H-2' e H-6'); 5,24 (sl; H-1 '); 4,33 (dd; $J$ 0,8 e 2,7 Hz; H-3"); 3,92 (m; H-4"); 3,49(m; H-5"). RMN de ${ }^{13} \mathrm{C}$ (75 MHz, CD $\mathrm{OD}, \delta): 179,68$ (C-4); 167,95 (C-7); 161,59 (C-5); 161,59 (C-4'); 161,60 (C-9); 159,08 (C-2); 134,74 (C-3); 131,89 (C-2', C-6'); 122,78 (C-1'); 116,53 (C-3', C-5'); 105,08 (C-10); 109,59 (C-1"); 100,52 (C-6); 95,25 (C-8); 88,04 (C-4"); 83,25 (C-2"); 78,59 (C-3"); 62,50 (C-5").

Quercetina 3- $\alpha$-raminosídeo (5). Sólido amarelo. EM (ESI) em $\mathrm{m} / z 455[\mathrm{M}+\mathrm{Na}]$. RMN de ${ }^{1} \mathrm{H}\left[300 \mathrm{MHz}, \mathrm{CD}_{3} \mathrm{OD}, \delta, J(\mathrm{~Hz})\right]: 7,34$ (m; H-2'/H-6'); 6,91 (d; J 7,6 Hz; H-5' ); 6,35 (sl; H-8); 6,18 (sl; H-6); 5,35 (sl; H-1"); 4,23 (m; H-2"); 3,77 (dd; J 7,5 Hz; J 1,5 Hz; H-3"); 3,42 (m; H-5"); 3,35 (m; H-4"); 0,94 (d; J 5,8 Hz ; H-6"). RMN de ${ }^{13} \mathrm{C}$ (75 MHz, CD $\left.\mathrm{OD}, \delta\right): 179,54$ (C-4); 166,35 (C-7); 163,01 (C-5); 158,45 (C-2); 158,24 (C-9); 149,78 (C-4'); 146,36 (C-3'); 136,14 (C-3); 122,95 (C-1'); 122,86 (C-6'); 116,40 (C-2'); 116,96 (C-5'); 105,71 (C-10); 103,49 (C-1"); 100,04 (C-6); 94,89 (C-8); 73,26 (C-4"); 72,11 (C-3"); 71,99 (C-2"); 71,87 (C-5"); 17,62 (C-6").

Alcanoatos de fitila (3). Óleo. EM-IE (30eV); m/z (\%) 257(100); 285 (25); 313(13); e 341(5); 369 (2) 648 (1); m/z 676 (5); m/z 704 (3) e $m / z, 732$ (2). RMN de ${ }^{1} \mathrm{H}\left[300 \mathrm{MHz}, \mathrm{CDCl}_{3}, \delta, J(\mathrm{~Hz})\right]: 5,31(\mathrm{~m}$; H-2); 4,60 (d; 7,2 Hz; H-1); 2,29 (t; 7,5; H-4); 1,70 (s; H-20); RMN de ${ }^{13} \mathrm{C}$ (75 MHz, $\left.\mathrm{CDCl}_{3}, \delta\right): 61,43$ (C-1); 118,43 (C-2); 142,80 (C-3); 39,86 (C-4); 25,02 (C-5); 36,88 (C-6); 32,90 (C-7); 37,54 (C-8); 24,70 (C-9); 37,60 (C-10); 32,91 (C-11); 37,66 (C-12); 24,70 (C-13); 39,61 (C-14); 28,22 (C-15); 22,84 (C-16); 22,92 (C-17); 19,93 (C-18); 19,59 (C-19); 16,59 (C-20); 174,24 (C-1'); 34,66 (C-2'); 14,32 ( $\left.\underline{\mathrm{CH}}_{3}\right)$.

\section{RESULTADOS E DISCUSSÃO}

A partir da fração AcOEt obtida do extrato metanólico das folhas de T. guianensis foram isolados, por técnicas cromatográficas, uma mistura de norisoprenóides diastereoisoméricos $\mathbf{1}$ e $\mathbf{2}$, além dos flavonóides, 3, 4 e 5 (Figura 1).

A fórmula molecular $\mathrm{C}_{19} \mathrm{H}_{30} \mathrm{O}_{8}$ foi proposta para 1 e 2 , com base no espectro de massas (ESI-EM) pela presença dos íons pseudomoleculares $m / z, 409[\mathrm{M}+\mathrm{Na}]^{+} \mathrm{e} \mathrm{m} / z, 387[\mathrm{M}+\mathrm{H}]^{+}$, aliado aos dados de $\mathrm{RMN}$ de ${ }^{1} \mathrm{H} \mathrm{e}{ }^{13} \mathrm{C}$ (incluindo experimento DEPT). A identificação 
<smiles>CC1=CC(=O)CC(C)(C)C1(O)/C=C/C(C)C</smiles><smiles>CC1=CC(=O)CC(C)(C)C1(O)/C=C/C(C)C</smiles><smiles>[R]Oc1c(C2C=CC(O)=C([R])C2)oc2cc(O)cc(O)c2c1=O</smiles>

$3 \mathrm{R}=\alpha-\mathrm{Rha}, \mathrm{R}_{1}=\mathrm{H}$

$4 \mathrm{R}=\alpha-\mathrm{Ara}, \mathrm{R}_{1}=\mathrm{H}$

$5 \mathrm{R}=\alpha-\mathrm{Rha}, \mathrm{R}_{1}=\mathrm{OH}$

Figura 1. Substâncias isoladas de Tapirira guianensis

destas substâncias, incluindo a determinação das configurações relativa e absoluta das mesmas, pode ser realizada pelas análises detalhada dos espectros de RMN e das curvas de dicroísmo circular. $\mathrm{O}$ espectro de $\mathrm{RMN}{ }^{1} \mathrm{H}$ evidenciou a presença de um multipleto em $\delta_{\mathrm{H}} 5,86$, além de dois duplos dupletos em $\delta_{\mathrm{H}} 5,97(15,0 ; 1,0 \mathrm{~Hz})$ e $\delta_{\mathrm{H}}$ $5,73(15,0 ; 7,0 \mathrm{~Hz})$ sugerindo a presença de ligação dupla com configuração $E$. Neste espectro também foi possível observar a presença de dois quartetos de dupletos, em $\delta_{\mathrm{H}} 4,53(7,8$ e $3,9 \mathrm{~Hz})$ e 4,42 $(6,9$ e $3,6 \mathrm{~Hz}$ ) atribuíveis aos hidrogênios oximetínicos $(\mathrm{H}-9)$. Além destes foram verificados dois dupletos em $\delta_{\mathrm{H}} 4,34(J=8,0 \mathrm{~Hz})$ e $4,27(J=8,0$ $\mathrm{Hz}$ ), indicativos de hidrogênios anoméricos da $\beta$-glicose. A presença de uma unidade glicosídica na estrutura foi confirmada pela análise dos demais sinais de $\mathrm{RMN}{ }^{1} \mathrm{H}$ e $\mathrm{RMN}{ }^{13} \mathrm{C}$ e pela comparação com os dados da literatura. ${ }^{8}$

A presença de dois hidrogênios oximetínicos (H-9) constatada pelo espectro de $\mathrm{RMN}{ }^{1} \mathrm{H}$ contribuiu para sugerir que a fração se tratava de uma mistura binária diastereisomérica. Foi possível determinar por comparação dos valores de integração $(1: 0,97)$ dos sinais de H-9 $\left(\delta_{H} 4,42 ; \delta_{H} 4,53\right)$ que as substâncias se encontram, aproximadamente, na razão 1:1. Devido às pequenas diferenças nos deslocamentos químicos da maioria dos sinais de $\mathrm{RMN}{ }^{1} \mathrm{H}$ dos componentes da mistura, a atribuição destes sinais para cada substância foi bastante dificultada. Porém a análise dos resultados do experimento TOCSY 1D possibilitou diferenciar de forma inequívoca os sinais de cada uma das substâncias da mistura, facilitando a interpretação dos espectros de RMN bidimensionais.

Pela análise do espectro de $\mathrm{RMN}{ }^{13} \mathrm{C}$ considerando deslocamento químico e intensidade dos sinais pôde-se evidenciar a presença dos sinais dos carbonos de dois sistemas carbonílicos $\alpha, \beta$-insaturados $\left(\delta_{\mathrm{C}} 201,22 ; \delta_{\mathrm{C}} 127,16 ; \delta_{\mathrm{C}} 127,10 ; \delta_{\mathrm{C}} 167,29\right)$. Foram verificados também sinais para uma ligação dupla $\left(\delta_{C} 131,52\right.$ e $\left.\delta_{C} 135,26\right)$, além de sinal de carbono oxigenado não hidrogenado $\left(\delta_{C} 80,01\right)$ e quatro carbonos metílicos $\left(\delta_{\mathrm{C}} 21,18 ; \delta_{\mathrm{C}} 23,42 ; \delta_{\mathrm{C}} 24,69\right.$ e $\left.\delta_{\mathrm{C}} 19,56\right)$. A análise das correlações observadas no espectro HMQC permitiu atribuir para os carbonos metílicos os respectivos sinais de RMN ${ }^{1} \mathrm{H}$. Pela multiplicidade dos sinais, pôde-se concluir que pelo menos dois grupos metílicos se encontram diretamente ligados a carbonos não hidrogenados.

As correlações verificadas no espectro $\mathrm{HMBC}$ entre os sinais em $\delta_{\mathrm{H}} 1,29(\mathrm{C}-10)$ com os sinais em $\delta_{\mathrm{C}} 77,29$ e 135,26 possibilitaram estabelecer a conectividade de três carbonos (C-8, C-9 e C-10). A partir deste espectro também pôde ser verificada a correlação entre os sinais de $\mathrm{H}-4\left(\delta_{\mathrm{H}} 5,87\right)$ e os sinais dos carbonos em $\delta_{\mathrm{C}} 19,56$ e 80,01. Estes dados aliados às correlações observadas entre os sinais de $\mathrm{H}-2$ equatorial $\left(\delta_{\mathrm{Heq}} 2,60\right)$ e axial $\left(\delta_{\mathrm{H} a \mathrm{x}} 2,16\right)$ e os sinais em $\delta_{\mathrm{C}} 23,42 ; 42,43$; 80,01 e 201,22 auxiliaram a se estabelecer a conectividade entre os carbonos do anel de seis membros de $\mathbf{1}$. Finalmente, o espectro HMBC possibilitou inferir a posição da glicosilação pela correlação entre o hidrogênio anomérico $\left(\delta_{\mathrm{H}} 4,34\right)$ e o respectivo carbono carbinólico C-9 $\left(\delta_{C} 77,29\right)$. A atribuição dos deslocamentos químicos dos carbonos e hidrogênios para 2 foi realizada de forma análoga.

A configuração absoluta do centro estereogênico C-6 foi determinada com base nas análises das curvas de dicroísmo circular de $\mathbf{1}+\mathbf{2}$ e naquelas de compostos modelos, cujas configurações absolutas estão bem estabelecidas na literatura, como para o (6S)-hidroxi-3oxo- $\alpha$-ionol. ${ }^{9}$ Estes compostos caracterizam-se como $6 R$ quando apresentam um sinal de efeito Cotton negativo na região de $240 \mathrm{~nm}$, e $6 S$ quando apresentam um efeito positivo na mesma região espectral. Como foi observado para 1+2 um efeito Cotton positivo em $240 \mathrm{~nm}$ $(\varepsilon \Delta=20,5)$, causado pela helipticidade entre a enona e a ligação dupla em C-7, pôde-se concluir que $\mathbf{1}$ e $\mathbf{2}$ apresentam configuração $6 S .{ }^{8}$ A determinação da configuração do centro estereogênico C-9 foi baseada na comparação dos valores dos deslocamentos químicos destes carbonos com aqueles descritos na literatura para o 3- $\alpha$-ionol$\beta$-glicopiranosídeo, cuja configuração absoluta foi determinada após análises dos produtos da reação com reagentes de Mosher. ${ }^{9}$ Assim, estabelece-se a configuração 9S para os carbonos (C-9) que apresen$\operatorname{tam} \delta \sim 74$ e $9 R$ para aqueles com $\delta \sim 77$. Logo, a substância 1 deve ter a configuração absoluta $6 S, 7 E, 9 R$ e 2 a configuração $6 S, 7 E, 9 S .{ }^{10}$

Os flavonóides 3-5 foram identificados pela análise detalhada dos dados de $\mathrm{RMN}{ }^{1} \mathrm{H}, \mathrm{RMN}{ }^{13} \mathrm{C}$ e pela comparação com os dados daqueles já descritos na literatura para flavonóides glicosilados. ${ }^{11}$ Baseado nas diferenças dos deslocamentos químicos dos carbonos C-2 e C-3 quando comparadas ao da aglicona, foi possivel identificar os flavonóides como sendo canferol 3- $\alpha$-raminosídeo (3), canferol 3- $\alpha$-arabinofuranosídeo (4) e quercetina 3- $\alpha$-raminosídeo (5), também conhecida como quercetrina.

Os espectros de $\mathrm{RMN}{ }^{1} \mathrm{H}$ e $\mathrm{RMN}{ }^{13} \mathrm{C}$ de 6-8 (Figura 2) apresentaram evidências compatíveis com um derivado do fitol. No espectro de $\mathrm{RMN}{ }^{1} \mathrm{H}$ puderam ser identificados sinais característicos do fitol, tais como do H-2 $(\delta 5,31)$, além do dupleto em $\delta_{\mathrm{H}} 4,60(\mathrm{~J}=$ $7,2 \mathrm{~Hz}$ ) referente aos hidrogênios oximetilênicos $2 \mathrm{H}-1$. O valor do deslocamento químico destes hidrogênios foi indicativo de que 6-8 se tratavam de derivados esterificados do fitol. Foram observados no espectro de $\mathrm{RMN}$ de ${ }^{13} \mathrm{C}$, além dos sinais relativos aos carbonos da ligação dupla $\left(\delta_{C} 142,80\right.$ e $\left.\delta_{C} 118,43\right)$, um sinal de carbono não hidrogenado $\left(\delta_{C} 174,24\right)$, típico de um carbono acílico de éster. Esta inferência pode ser confirmada pelo espectro de absorção no IV, pela presença das bandas de estiramento em 1724 e em $1203 \mathrm{~cm}^{-1}$. No espectro de RMN de ${ }^{13} \mathrm{C}$ foi observado o sinal de carbono metilênico oxigenado em $\delta_{\mathrm{C}} 61,43$, mais desprotegido que o correspondente $\mathrm{C}-1$ do fitol, devido ao efeito da acilação. Os deslocamentos químicos dos carbonos observados para a maioria dos demais sinais foram similares aos do fitol. Entretanto, sinais típicos de uma cadeia alquílica linear saturada foram observados no espectro. Pela análise por CG-EM dos ésteres metílicos obtidos da reação de transesterificação dos componentes da fração foi possível concluir que a mistura 6-8 era constituída por pelo menos três ésteres metílicos majoritários. Este dado foi revelado pela presença de três picos, correspondentes aos íons moleculares $m / z$ 270; 298 e 326 . Estes íons moleculares e o padrão de fragmentação destes ésteres metílicos permitem propor que os ácidos hexadecanóico, octadecanóico e eicosanóico estão esterificados com o fitol na mistura 6-8.

A mistura dos ácidos graxos esterificados com $\alpha$-amirina (9-12) foi identificada a partir dos dados observados nos espectros de RMN ${ }^{1} \mathrm{He} \mathrm{RMN}{ }^{13} \mathrm{C}$, que se mostraram compatíveis com os observados para o derivado de $\alpha$-amirina esterificado com ácido graxo de cadeia longa. ${ }^{12} \mathrm{~A}$ análise dos espectros de massas dos ésteres metílicos obtidos após transesterificação destes compostos possibilitou identificar os 


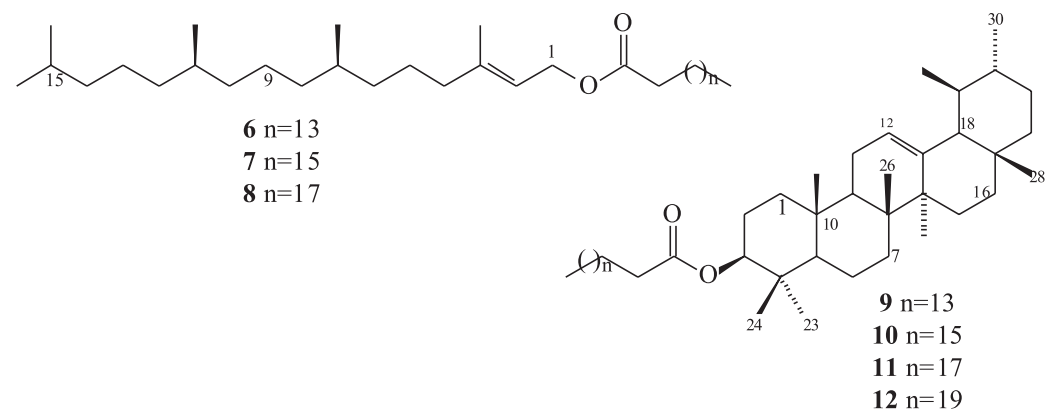

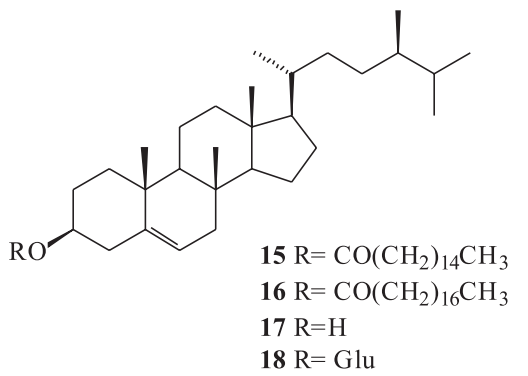

Figura 2. Terpenóides de T. guianensis

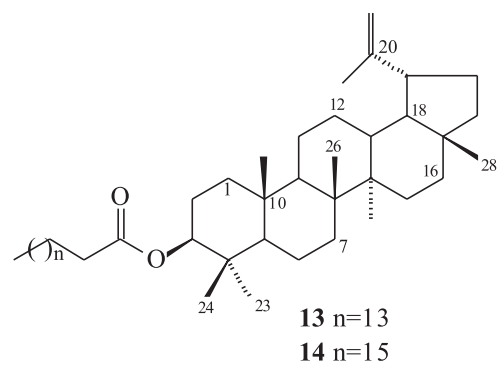<smiles>CC(C)C(C)CC[C@H](C)C1CCC2C1(C)CCC1C3(C)CCC(=O)C=C3CCC12C</smiles><smiles>C=C(CC[C@H](C)C1CC[C@H]2C1(C)CCC13CC14CCC(O)C(C)(C)C4CCC23C)C(C)C</smiles>

ácidos graxos esterificados com o triterpeno como sendo os ácidos hexadecanóico, octadecanóico, eicosadecanóico e dodeicosanóico. Pela área dos picos observados TIC constatou-se que os derivados dos ácidos C-16 e C-18 eram os majoritários. Assim, pôde-se concluir que a fração era constituída de derivados de quatro ácidos de cadeia longa, hexadecanoato (9), octadecanoato (10), eicosanoato (11) e dodeicosanoato (12) de $\alpha$-amirinila. Para a identificação dos constituintes da mistura dos alcanoatos de fitila, lupeol e sitosterila considerou-se principalmente o espectro de $\mathrm{RMN}{ }^{13} \mathrm{C}$.

Usando a metodologia empregada para identificação de substâncias terpenoídicas em mistura, ${ }^{13}$ foi possível identificar a mistura 13-16 a partir das intensidades e multiplicidades dos sinais de RMN ${ }^{13} \mathrm{C}$ (incluindo experimento DEPT) dos carbonos das ligações duplas e carbonos oxigenados das diferentes substâncias. Assim, os sinais $\delta_{C}$ 150,$56 ; 109,24$ e 80,38 foram considerados pertencentes ao esqueleto terpenoídico das substâncias 13-14 e os sinais em $\delta_{C} 139,49 ; 122,39$ e 73,47 das substâncias 15-16. Após derivatização da mistura por meio da reação de transesterificação e obtenção dos ésteres metílicos correspondentes, estes foram analisados por CG-EM. No cromatograma foram registrados dois picos majoritários com diferentes tempos de retenção, e cada um deles originou um espectro de massas cujos respectivos íons moleculares em $\mathrm{m} / \mathrm{z} 298$ e em 270 e fragmentos são correspondentes ao estearato de metila e ao palmitato de metila, respectivamente. Estes resultados permitem sugerir que os compostos da mistura se encontram esterificados, principalmente com os ácidos palmítico e esteárico.

Este é o primeiro relato da ocorrência dos derivados glicosilados de norisoprenóides (megastimanos), de alcanoatos de fitila, bem como de terpenos esterificados com ácidos graxos em Anacardiaceae. Previamente só foram descritos a presença de triterpenos livres na família. ${ }^{2}$ Os flavonóides $\mathbf{3}$ e $\mathbf{5}$ foram previamente encontrados em Sclerocarya birrea ${ }^{14}$ no entanto, esta é a primeira descrição da ocorrência de $\mathbf{4}$ em Anacardiaceae.

\section{AGRADECIMENTOS}

À CAPES, ao CNPq, IMSEAR e à FAPESB pelas bolsas e apoio financeiro e à Profa. Dra. L. Beltramini (UFSCar) pela obtenção das curvas de dicroísmo circular.

\section{REFERÊNCIAS}

1. Vogl, O.; Mitchell, J. D.; Pure Appl. Chem. 1996, 33, 1581; Correia, S. J.; David, J. M.; David, J. P.; Quim. Nova 2006, 29, 1287; Evans, F. J.; Schmidt, R. J.; Planta Med 1980, 38, 289.

2. David, J. M.; Chavez, J. P.; Chai, H. B.; Pezzuto, J. M.; Cordell, G. A.; J. Nat. Prod. 1998, 61, 287.

3. Lorenzi, H.; Árvores Brasileiras. Instituto Plantarum, Ed. Nova Odessa: São Paulo, 2002.

4. Goetz, G. M.; Lepoittevin, J. P.; Bioorg. Med. Chem. Lett. 1999, 9, 1141.

5. Correia, S. J.; David, J. M.; David, J. P.; Chai, H.-B.; Pezzuto, J. M.; Cordell, G. A.; Phytochemistry 2001, 56, 781.

6. Matsuda, N.; Isawa, K.; Kikuchi, M.; Phytochemistry 1997, 45, 777.

7. Schuchardt, U.; Sercheli, R.; Vargas, R. M.; J. Braz. Chem. Soc. 1998, 9, 199.

8. Pabst, A.; Barron, D.; Sémon, E.; Schreier, P.; Phytochemistry 1992, 31, 1649.

9. Çalis, I.; Kuruüzüm-Uz, A.; Lorenzetto, P. A.; Rüedi, P.; Phytochemistry 2002, 59, 451 .

10. Peng, Z. F.; Strack, D.; Baumert, A.; Subramaniam, R.; Goh, N. K.; Chia, T. F.; Tan, S. N.; Chia, L. S.; Phytochemistry 2003, 62, 219; Fossen, T.; Larsen, A.; Kiremire, B. T.; Andersen, Ø. M.; Phytochemistry 1999, 51, 1133; Guo, J.; Yu, D.; Xu, L.; Zhu, M.; Yang, S. L.; Phytochemistry 1998, $48,1445$.

11. Agrawal, P. K.; Thakur, R. S.; Bansal, M. C.; Foo, L. Y.; Markham, K. R.; Porter, K. J. Em Carbon-13 NMR of Flavonoids; Agrawal, P. K., ed.; Elsevier: Amsterdam, 1989.

12. Mahato, S. B.; Kundu, A. P.; Phytochemistry 1994, 37, 1517; Chávez, J. P.; Dos Santos, I. D.; Cruz, F. G.; David, J. M.; Phytochemistry 1996, 41,941 .

13. Gallegos, R. S.; Roque, N. F.; Quim. Nova 1990, 13, 278.

14. Braca, A.; Politi, M.; Sanago, R.; Sanou, H.; Morelli, I.; Pizza, C.; De Tommasi, N.; J. Agric. Food Chem. 2003, 51, 6689. 